Sebastian Ley ${ }^{1}$, Bermet Estebesova ${ }^{2}$

貫 ley@gmx.net

in. www.linkedin.com/in/sebastian-prof-dr-ley-92b4a97b/

\title{
Editorial
}

\section{Thoracic imaging: course report}

Cite as: Ley S, Estebesova B.

Thoracic imaging: course report. Breathe 2019; 15: 4-6.

\section{Overview}

Clinical symptoms of patients with different pulmonary diseases can be quite similar. Further diagnostic work-up includes various tests and most often some kind of imaging. While in academic centres these imaging studies are being interpreted by a radiologist in a reasonable time, this might not be the case in all settings. Therefore, respiratory physicians are highly interested in getting an in-depth knowledge of interpretation of radiology imaging studies.

The European Respiratory Society (ERS) provides courses on imaging and also during the ERS International Congress imaging has a space in the programme. However, most courses are specialised courses, not covering the broad field of imaging from the basics to high-level diagnosis in a systematic approach. Furthermore, most courses are organised as lectures with little or no possibility for the audience to interact with the speaker or even discuss cases in detail.

To overcome these restrictions, the ERS organised a 3-day course on thoracic imaging. This was the fourth time the course took place, after courses in 2012, 2013 and 2015. The course took place on October 25-27, 2018, again in the beautiful city of Barcelona, Spain. As in the previous years, the course was fully booked, with 80 participants on site. For the first time, the lectures were broadcasted as online lectures, where another 60 participants registered.
Most of the participants were clinicians from a wide variety of European countries and some came from Arabic and Asian countries. Some of the participants were economically supported by the ERS (totally or partially), after evaluation of the applicants. The clinical level of the participants ranged from residents up to senior physicians.

The course was structured based on two main components: 1) integrating frontal lectures with an interactive voting system, and 2) interactive, break-out sessions (workshops). For workshops, participants were divided into smaller groups, each able to attend all workshops. Within the seminars, individual cases were discussed in an interactive manner with enough time to discuss various aspects and challenges of imaging findings. Each seminar was held by an expert in chest radiology and a senior ERS pulmonologist. Based on the feedback of the participants from previous years, further improvements in structure and content of the lectures and workshops were integrated.

The curriculum covered all aspects of thoracic imaging: obstructive, infectious, occupational, interstitial lung disease, small and large airway diseases, mediastinal and vascular/right heart pathologies. The main imaging modalities of radiography, computed tomography and magnetic resonance imaging were addressed within the course. The participants were highly motivated to learn about all the different aspects of imaging,

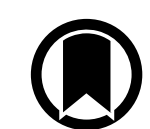

\section{CrossMark}

CCERS 2019
@ERSpublications

Faculty and a delegate of an ERS course on thoracic imaging describe their experiences http://ow.ly/dYPa30n3wXj 


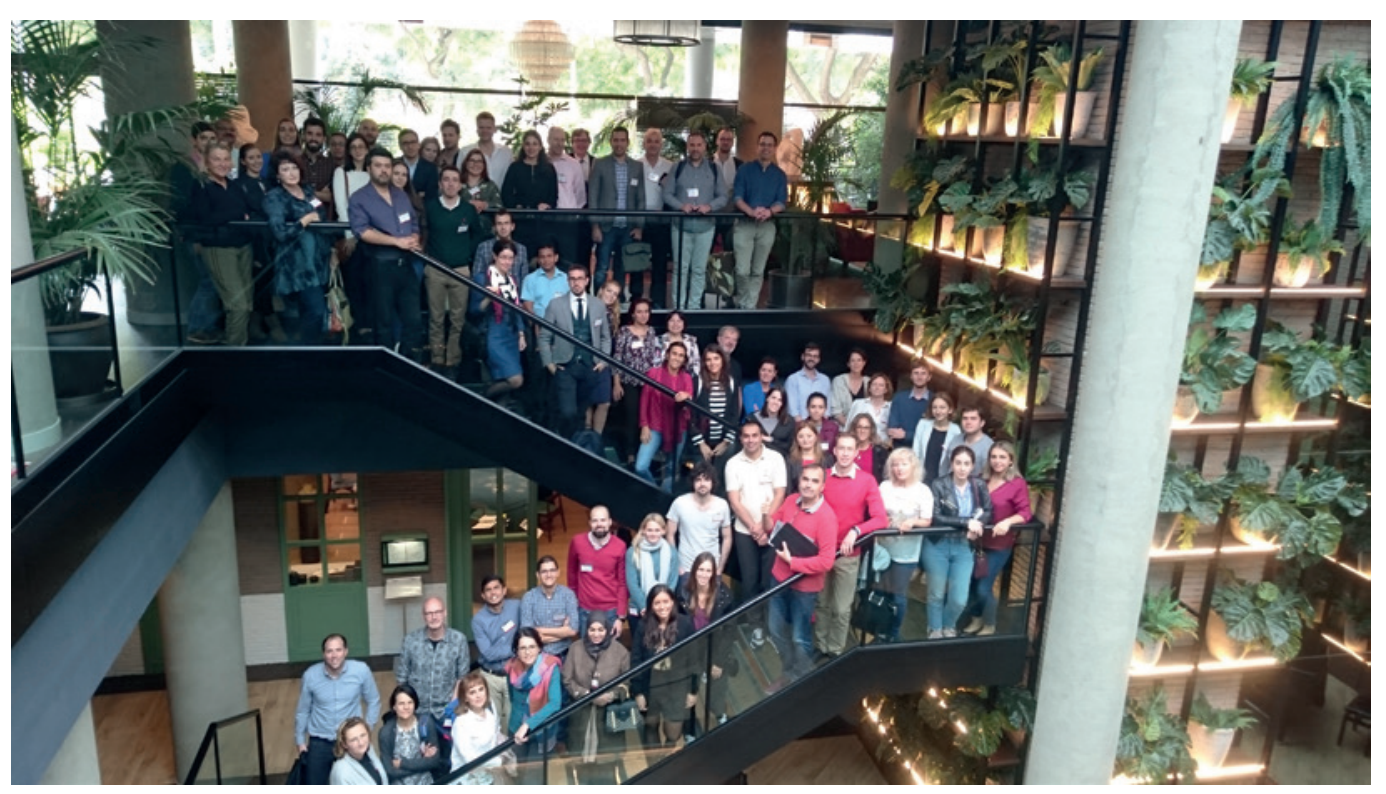

and many questions were placed at the end of each presentation. As usual, the field of interstitial lung disease, namely the differentiation between various forms, was of especially high interest.

Internationally renowned chest radiologists and respiratory physicians were invited as speakers, and all participants had the chance to have close discussion with them about difficult cases during the lectures, seminars, coffee breaks and lunches. To further stimulate the discussion and exchange, an image interpretation session with cases from the audience was performed. With participants from Europe and non-European countries, a discussion about diagnostic and treatment protocols was stimulated. This discussion was especially motivated in the case presentation session, where 15 participants demonstrated their own cases with clinical and imaging findings. At this point, I would like to extend a big "thank you" for all the work put into the selection of interesting cases and preparation of the presentations.

In conclusion, the course was a great success and the ERS will continue providing education on imaging. The course format was well chosen, providing a structured, lecture-based approach to the various topics, with new information for the beginners and an update/refresh for the seniors. The interactive seminars were particularly interesting for the participants, as all questions on a topic could be discussed from a radiological and clinical perspective. This was again strongly positively mentioned by the participants in the evaluation of the course.

Last but not least, such a positive experience would have not been possible without the usual hard work and highly professional job of the ERS staff.

\section{Bermet Estebesova, Kyrgyzstan, a participant}

Thoracic imaging methods are an integral part of a comprehensive examination of most patients with respiratory diseases, which is sometimes crucial for the timely correct diagnosis and choice of adequate therapy. Today, when we have a large variety of interstitial diseases, it is very important to clearly know radiographic and computed tomography signs of diseases.

The ERS Thoracic Imaging course included all important topics and was very interesting for early career specialists as well as for more experienced participants, physicians and radiologists. It was a very good opportunity not only to refresh basic knowledge, but also to advance with the most recent studies in the domain of chest medicine.

Starting with the simplest and most frequently used technique, chest radiography, we talked about the technique for good visualisation of the thoracic cavity and the importance of using a systematic approach for interpretation. We moved on to more informative computed tomography or magnetic resonance imaging, which are very useful in more difficult cases. We learnt that we need more detailed visualisation, sometimes endoscopic ultrasonography or endobronchial ultrasonography, for the evaluation of mediastinum. All the topics included in the course programme were presented in a very accessible and comprehensible way with many clinical cases, where we were shown all the radiological signs and nuances of certain respiratory diseases. For example, it is very important to be able to differentiate between bronchiolitis, cysts and mosaic patterns that 
result from impairments of the airways or lung parenchyma in occupational, infection or cardiovascular diseases. In the workshops we had the opportunity to practise and discuss all points that we wanted to clarify with the speakers. Their kindness and modesty were very fruitful. Furthermore, we had the opportunity to practise ultrasound of the chest and train with devices for ultrasound-controlled biopsy, which was one the most interesting parts of the course. During all the workshops, we had hot discussions on difficult cases that needed a more detailed characterisation of radiological signs.

The atmosphere was very friendly. The breaks were nicely arranged and allowed for a moment "to take a breath" and start over.

\section{Affiliations}

\section{Sebastian Ley ${ }^{1}$, Bermet Estebesova ${ }^{2}$}

${ }^{1}$ Diagnostic and Interventional Radiology, Chirurgisches Klinikum München Süd, Munich, Germany. ${ }^{2}$ National Center of Cardiology and Internal Medicine, Bishkek, Kyrgyzstan.

Conflict of interest

None declared. 